\title{
Prevalence of Erectile Dysfunction and its Associated Factors among Vietnamese Men with Type 2 Diabetes
}

\author{
Khoa Tuan Vo, ${ }^{1}$ Hoi Quoc Huynh, ${ }^{1}$ Nam Quang Tran, ${ }^{2}$ Aya Goto, ${ }^{3,4}$ Yuriko Suzuki, ${ }^{3,}{ }^{5}$ Khue Thy Nguyen 6 \\ ${ }^{1}$ Department of Endocrinology, People's Hospital 115, Vietnam \\ 2 Department of Endocrinology, University of Medicine and Pharmacy, Ho Chi Minh City, Vietnam \\ ${ }^{3}$ Department of Public Health, Fukushima Medical University School of Medicine, Japan \\ ${ }^{4}$ Takemi Program in International Health, Harvard School of Public Health, Japan \\ ${ }^{5}$ Department of Adult Mental Health, National Institute of Mental Health, National Center of Neurology and Psychiatry, Japan \\ ${ }^{6} \mathrm{Ho}$ Chi Minh City Medical Association, Vietnam
}

\begin{abstract}
Objective. This study sought to estimate the prevalence of erectile dysfunction (ED) and to ascertain risk factors among Vietnamese men with diabetes.

Methodology. One hundred and fifty one diabetic patients were recruited from the People's Hospital 115 between August 2011 and March 2012. They were asked to complete a self-administered questionnaire that included the International Index for Erectile Function Questionnaire (IIEF-5) and the World Health Organization (WHO) - Five Wellbeing Index. Medical history, anthropometric measures, and laboratory test results were recorded. ED was classified based on the IIEF-5 total score.
\end{abstract}

Results. Of the 151 diabetic men, 135 (89\%) completed the IIEF-5. The overall prevalence of ED was 84\%; majority $(65 \%)$ were classified as mild to moderate, while $10 \%$ were considered severe. The prevalence of ED was higher among those with peripheral neuropathy [adjusted odds ratio (OR) 5.22, 95\% confidence interval $(\mathrm{Cl}) 1.14-21.89$ ] and longer duration of diabetes (OR 1.11, 95\% Cl 1.00-1.24).

Conclusion. ED represents a prevalent major complication among men with type 2 diabetes. The presence of peripheral neuropathy and long duration of diabetes may be useful indicators of ED.

Key words: diabetes mellitus, erectile dysfunction, Vietnam, peripheral neuropathy, questionnaire

\section{INTRODUCTION}

Erectile dysfunction has been a taboo topic in Asian countries, but related research has been increasing recently. The reported prevalence of ED in Asian countries varies greatly, from $2 \%$ to $88 \% .^{1}$ A recent meta-analysis summarized age-specific prevalence rates as follows: $15 \%$ for ages 20 to $29,30 \%$ for 30 to $39,41 \%$ for 40 to $49,54 \%$ for 50 to 59 , and $70 \%$ for 60 to $69 .{ }^{2}$ The rate is even higher among men with chronic diseases including diabetes, according to an international study including two Asian countries. ${ }^{3}$ The prevalence of ED among men with diabetes also varies widely, from $35 \%$ to $90 \%{ }^{4}$ The risk of developing ED is about 3 times higher in diabetic men compared to those who do not have diabetes. ${ }^{5,6}$ Most of the data on ED in diabetics are from non-Asian countries. In a few studies conducted in Vietnam, the frequency of ED was found to be $40 \%$ in the general population over 40 years of age, and $65.3 \%$ in those with type 2 diabetes.7.8 While these locally published studies reflect an increasing

e-ISSN 2308-118x

Printed in the Philippines

Copyright (C) 2014 by the JAFES

Received January 1, 2014. Accepted April 29, 2014. awareness of ED in the country, practical recommendations for diabetes specialists are limited.

In this study, the International Index for Erectile Function Questionnaire (IIEF-5), an internationally accepted and practical measure, was used to estimate the prevalence of ED and investigate its associated factors among Vietnamese men with diabetes visiting a general public hospital in Ho Chi Minh City. The aim was to present recommendations for Vietnamese diabetes specialists that can be easily applied in their clinical practice.

\section{METHODOLOGY}

We conducted a cross-sectional investigation between August 2011 and March 2012 at the People's Hospital 115 in Ho Chi Minh City, Vietnam. Formerly a military clinic, it is now a tertiary university-affiliated general hospital with 1500 beds. On the average, the hospital receives about 100 outpatients with diabetes per day. The subjects
Corresponding author: Khoa Tuan Vo, MD

Department of Endocrinology, People's Hospital 115

527 Su Van Hanh, Ward 12, District 10

Ho Chi Minh City, Vietnam

Tel. No.: +84-9-3776-3774

Fax No.: +84-8-3865-5193

E-mail: tkhoa.vo@gmail.com 
were recruited by convenience sampling from an outpatient clinic. Criteria for inclusion were male gender, diagnosed with type 2 diabetes, age 30 to 65 years and currently married. Exclusion criteria were concomitant chronic diseases such as kidney, liver or chronic obstructive pulmonary disease; and the inability to complete a self-administered questionnaire due to eye or extremity disabilities.

During the study period, the affiliated physicianresearchers recruited 151 patients that they encountered for a total of 34 days. Survey days were selected according to the researchers' schedule. Efforts were made to ensure patient privacy while accomplishing the questionnaires. Male physicians were in attendance.

We used IIEF-5 to assess erectile dysfunction. Extracted from the original 15-item IIEF, this 5-item measurement is standardized and now widely used for the diagnosis and classification of ED severity. ${ }^{9}$ The 5 items assess erection confidence, erection firmness, maintenance frequency, maintenance ability and intercourse satisfaction in the past 6 months. The total score was the basis for classification of ED: no dysfunction (22-25), mild dysfunction (17-21), mild to moderate dysfunction (12-16), moderate dysfunction (811) and severe dysfunction (5-7). Since a Vietnamese language version was not available, we went through a rigorous back-translation procedure to ensure an accurate and culturally adequate translation. Initially, 3 Vietnamese native speakers independently translated the original English IIEF-5 into Vietnamese, then collectively discussed how to develop the first consensus version. Secondly, 2 English native speakers back-translated the first version into English. In the third step, we compared the original and back-translated English versions to finalize the Vietnamese language version. For the fourth step, we conducted a test-retest face-to-face survey among 20 volunteer diabetic patients with an interval of 4 weeks to examine reliability. Twenty-five patients were included in the first interview, but only 20 patients returned for the second. Spearman's correlation coefficient of the two scores was 0.56 ; the mean difference in score was 0.4 , with a standard deviation of 4.8 . In addition, we paid careful attention to the order and layout of questions to make the questionnaire easy to fill out by Vietnamese patients.

Other survey items included age, socioeconomic status (occupation and education level), lifestyle (smoking, alcohol consumption, physical activity), diabetes treatment history, family history of diabetes, medical history (stroke, cardiovascular disease and foot ulcer), and mental state as measured by the WHO-Five Well-being Index. ${ }^{10}$ Anthropometrics included height $(\mathrm{m})$, weight $(\mathrm{kg})$ and waist circumference $(\mathrm{cm})$.

Patients were diagnosed with diabetic peripheral neuropathy if they showed at least one abnormal result in any of 3 tests: superficial pain testing, light touch perception and vibration sensation using a $128-\mathrm{Hz}$ tuning fork. For sensory testing, the patient was required to close his eyes and was given a sample test on the forearm. The patient was then asked about the characteristics of the sensation. He was then asked to identify the sensation experienced upon application of the test at the dorsum of the first toe, and the plantar aspect of the distal phalanx of the first, third, and fifth toe of each foot. The test was defined abnormal when no sensation was elicited. ${ }^{11}$ For vibratory testing, a $128-\mathrm{Hz}$ tuning fork was placed on the bony prominences at the dorsum of the big toe. The patient was asked to say when he began to sense a decrease in vibration. The tuning fork was then moved to the dorsal aspect of the distal phalanx of the examiner's thumb. The time (s) was estimated when vibration sensation diminished beyond the examiner's perception. The abnormal value was between 0 and 10 seconds. ${ }^{12}$

The presence of retinopathy was detected by fundus examination. Peripheral vascular disease was assessed by palpation of the dorsalis pedis pulse and/or Doppler ultrasonography. Autonomic neuropathy was evaluated by taking the patient's pulse rate at rest (more than 100 beats per minute) and/or orthostatic blood pressure gradient (more than $20 \mathrm{mmHg}$ ).

Blood samples were drawn and analyzed for fasting blood glucose, hemoglobin A1c (HbA1c), triglycerides, lowdensity lipoprotein (LDL) cholesterol, high-density lipoprotein (HDL) cholesterol, total cholesterol, testosterone, follicle-stimulating hormone (FSH) and luteinizing hormone (LH). Urine analysis including microalbuminuria was also performed.

Data were analyzed by Stata software version 12.0. Statistical significance was considered at $\mathrm{p}<0.05$. Odds ratios of ED defined as an IIEF-5 score of 21 or lower for each survey item were calculated using logistic regression model. The first analyses estimated crude OR for each item listed in Tables 1 to 3, with ED as a dependent variable. The second multivariate analysis included significant factors in the first analyses because of potential confounding effects.

The study protocol was reviewed and approved by the Scientific Research Committee of People's Hospital 115. All participants gave oral informed consent. Considering cultural adequacy, agreeing to and filling out a questionnaire was considered as giving consent to the survey.

\section{RESULTS AND DISCUSSION}

Of 151 patients invited, 135 completed the questionnaire, yielding a response rate of $89 \%$. The responders and the 16 non-responders did not differ in age and duration of diabetes. The mean age of the responders was 54 years (SD 7.9), while the median duration of diabetes was 7 years (range 5 to 24). Twenty-four percent were treated with 
insulin, and median $\mathrm{HbA} 1 \mathrm{c}$ was $7.9 \%$ (range 5.4 to 14.2 ). Using the IIEF-5, the overall prevalence of ED was $83.7 \%$, of which $34.1 \%$ were mild, $31.1 \%$ mild to moderate, $8.1 \%$ moderate, and $10.4 \%$ severe (Figure 1 ).

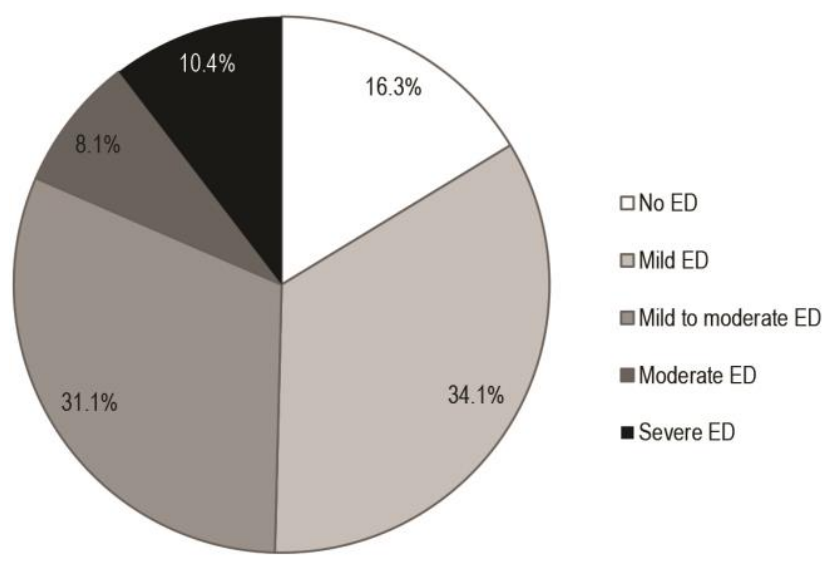

Figure 1. Distribution of erectile dysfunction among Vietnamese study participants with diabetes using the 5item International Index for Erectile Function Questionnaire. The total score was graded into 5 levels: no dysfunction (22 to 25 ), mild (17 to 21 ), mild to moderate (12 to 16), moderate (8 to 11$)$, and severe (5 to 7 ).

The risk for erectile dysfunction increased significantly with age (OR 1.06, 95\%CI 1.01-1.12) and duration of diabetes (OR 1.12, 95\%CI 1.01-1.25) (Tables 1 and 2). Similarly, the presence of diabetic peripheral neuropathy was found to be significantly associated with erectile dysfunction (OR 5.42, 95\%CI 1.20-24.39) (Table 2). None of the laboratory findings showed significant association (Table 3).
Our study found that the prevalence of ED in men with diabetes was $83.7 \%$. The percentage of men with diabetesassociated ED varies among studies due to age, duration of diabetes, study setting and measures used. ${ }^{4}$ Even with the same measurement, different cut-off values are suggested in different countries: an IIEF-5 score of 17 is applied in Korea, whereas 21 is applied in Italy. ${ }^{13,14}$ The prevalence of over $80 \%$ obtained in our study was in the upper end of the reported prevalence in Asian countries. ${ }^{2}$ A similar prevalence of $84.6 \%$ was reported among Chinese men with type 2 diabetes between 25 and 76 years of age. ${ }^{15}$

The observed high prevalence, despite cultural hesitation in the discussion of sexual issues in Vietnam, may be an overrepresentation caused by the selected study site, which was a tertiary referral hospital. Undetected ED cases, possibly from lack of physician awareness, may have left most ED cases untreated, and may have also increased its prevalence.

Age and duration of diabetes are important factors associated with ED as reported in previous studies and confirmed in our study. ${ }^{4,16-17}$ Disease duration was entered into a multivariate analysis with peripheral diabetic neuropathy and both remained significant. The association of peripheral diabetic neuropathy with ED has been reported in previous studies, including one from Japan by Sasaki and colleagues. ${ }^{18}$ The etiology of diabetic ED is multifactorial, with neuropathic, vasculopathic, hypogonadal and local pathologic changes.,6 Diabetic neuropathy includes autonomic and peripheral neuropathy; the former impairs relaxation of the smooth muscle of the corpus cavernosum, and the latter impairs sensory conduction from the shaft and glans of the penis to the reflexogenic erectile center. ${ }^{4}$

Table 1. Association of ED with baseline characteristics of study participants

\begin{tabular}{|c|c|c|c|c|c|}
\hline & \multirow{2}{*}{$\begin{array}{c}E D \\
n=113^{a}\end{array}$} & \multirow{2}{*}{$\begin{array}{c}\text { Non-ED } \\
n=22^{\mathrm{a}}\end{array}$} & \multicolumn{3}{|c|}{ Univariate analysis $^{\mathrm{b}}$} \\
\hline & & & Crude OR & $95 \% \mathrm{Cl}$ & $p$ value \\
\hline Mean age, yr & $54.7(7.6)$ & $50.7(8.6)$ & 1.06 & $1.01-1.12$ & 0.03 \\
\hline \multicolumn{6}{|l|}{ Occupation ${ }^{\mathrm{C}}$} \\
\hline Retired & $41(36.6)$ & $4(18.2)$ & 1.00 & & \\
\hline White collar & $40(35.7)$ & $10(45.4)$ & 0.67 & $0.26-1.75$ & 0.41 \\
\hline Blue collar & $31(27.7)$ & $8(36.4)$ & 0.67 & $0.26-1.68$ & 0.39 \\
\hline \multicolumn{6}{|l|}{ Education level } \\
\hline Illiterate, primary or secondary school & $18(16.1)$ & $2(9.1)$ & 1.00 & & \\
\hline High school & $48(42.8)$ & $8(36.4)$ & 1.31 & $0.51-3.38$ & 0.57 \\
\hline University/college & $46(41.1)$ & $12(54.5)$ & 0.58 & $0.23-1.46$ & 0.25 \\
\hline \multicolumn{6}{|l|}{ Smoking habit } \\
\hline Never & $41(36.3)$ & $7(31.8)$ & 1.00 & & \\
\hline Past & $26(23.0)$ & $4(18.2)$ & 1.03 & $0.27-3.87$ & 0.96 \\
\hline Current & $46(40.7)$ & $11(50.0)$ & 0.62 & $0.22-1.75$ & 0.37 \\
\hline \multicolumn{6}{|l|}{ Alcohol consumption } \\
\hline Never & $37(32.7)$ & $5(22.7)$ & 1.00 & & \\
\hline Sometimes & $50(44.3)$ & $11(50.0)$ & 0.55 & $0.18-1.70$ & 0.30 \\
\hline Regularly & $26(23.0)$ & $6(27.3)$ & 0.52 & $0.14-1.89$ & 0.32 \\
\hline \multicolumn{6}{|l|}{ Exercise } \\
\hline Never & $15(13.3)$ & $7(31.8)$ & 1.00 & & \\
\hline Sometimes & $26(23.0)$ & $3(13.6)$ & 3.37 & $0.77-14.80$ & 0.11 \\
\hline Regularly & $72(63.7)$ & $12(54.6)$ & 2.24 & $0.77-6.50$ & 0.14 \\
\hline
\end{tabular}


Table 2. Anthropometric and clinical characteristics of Vietnamese study participants with diabetes stratified by ED status

\begin{tabular}{|c|c|c|c|c|c|}
\hline & \multicolumn{2}{|c|}{ n (\%) ${ }^{\mathrm{a}}$ or median (range) } & \multicolumn{3}{|c|}{ Univariate analysis $^{b}$} \\
\hline & $\begin{array}{c}\text { ED } \\
(n=113)\end{array}$ & $\begin{array}{l}\text { Non-ED } \\
(n=22)\end{array}$ & Crude OR & $95 \% \mathrm{Cl}$ & $p$ value \\
\hline Body mass index, $\mathrm{kg} / \mathrm{m}^{-2}$ & $25.0(18.4,37.6)$ & $25.1(18.6,32.9)$ & 1.04 & $0.90-1.19$ & 0.59 \\
\hline Waist circumference, $\mathrm{cm}$ & $91(67,122)$ & $89.5(72,106)$ & 1.03 & $0.98-1.09$ & 0.23 \\
\hline Duration of diabetes, yr & $7.5(0.5,24)$ & $4(0.5,15)$ & 1.12 & $1.01-1.25$ & 0.03 \\
\hline Family history of diabetes & $44(38.9)$ & $12(54.6)$ & 0.53 & $0.22-1.33$ & 0.18 \\
\hline \multicolumn{6}{|l|}{ Past history } \\
\hline Cardiovascular disease & $4(3.5)$ & 0 & - & - & - \\
\hline Stroke & $3(2.7)$ & 0 & - & - & - \\
\hline Foot ulcer & $4(3.5)$ & 0 & - & - & - \\
\hline Hypertension & $76(67.3)$ & $12(54.6)$ & 1.71 & $0.68-4.32$ & 0.26 \\
\hline Systolic blood pressure, $\mathrm{mmHg}$ & $142(95,188)$ & $135(115,185)$ & 1.01 & $0.98-1.03$ & 0.55 \\
\hline Diatolic blood pressure, $\mathrm{mmHg}$ & $84(57,117)$ & $83.5(73,112)$ & 1.00 & $0.96-1.04$ & 0.92 \\
\hline \multicolumn{6}{|l|}{ Presence of chronic complications } \\
\hline Retinopathy & $17(15)$ & $5(22.7)$ & 0.60 & $0.20-1.85$ & 0.38 \\
\hline Peripheral neuropathy & $39(35.1)$ & $2(9.1)$ & 5.42 & $1.20-24.39$ & 0.03 \\
\hline Automatic neuropathy & $10(8.9)$ & $2(9.1)$ & 0.98 & $0.20-4.82$ & 0.98 \\
\hline Peripheral vascular disease & $3(2.7)^{\prime}$ & 0 & - & - & - \\
\hline Nephropathy & $26(28.3)$ & $2(11.8)$ & 2.96 & $0.63-13.83$ & 0.17 \\
\hline \multicolumn{6}{|l|}{ Antihypertensive medication } \\
\hline $\mathrm{ACEl}^{\mathrm{C}}$ & $60(79.0)$ & $10(83.3)$ & 0.75 & $0.15-3.77$ & 0.73 \\
\hline$A_{R B}{ }^{d}$ & $14(18.4)$ & $2(16.7)$ & 1.13 & $0.22-5.74$ & 0.88 \\
\hline $\mathrm{CCB}^{\mathrm{e}}$ & $27(35.5)$ & $5(41.7)$ & 0.77 & $0.22-2.67$ & 0.68 \\
\hline Diuretic & $9(11.8)$ & $1(8.3)$ & 1.48 & $0.17-12.84$ & 0.72 \\
\hline Beta blockers & $13(17.1)$ & $1(8.3)$ & 2.27 & $0.27-19.15$ & 0.45 \\
\hline \multicolumn{6}{|l|}{ Diabetes treatment } \\
\hline Diet alone & 0 & 0 & - & - & - \\
\hline Sulfonylurea & $69(61.6)$ & $15(68.2)$ & 0.75 & $0.28-1.99$ & 0.56 \\
\hline Metformin & $94(83.9)$ & $20(90.9)$ & 0.52 & $0.11-2.43$ & 0.41 \\
\hline Thiazolidinedione & 0 & 0 & - & - & - \\
\hline Dipeptidyl-peptidase- 4 inhibitor & 0 & 0 & - & - & - \\
\hline Insulin & $29(25.7)$ & $3(13.6)$ & 2.19 & $0.60-7.93$ & 0.23 \\
\hline Acarbose & 0 & 0 & - & - & - \\
\hline $\begin{array}{l}\text { WHO-Five Well-being Index } \\
\text { (Probable depressionf) }\end{array}$ & 59 (52.2) & $12(54.6)$ & 0.91 & $0.36-2.28$ & 0.84 \\
\hline \multicolumn{6}{|c|}{ 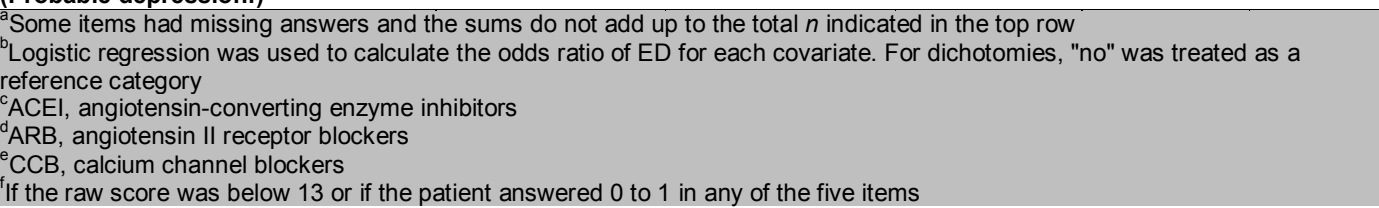 } \\
\hline
\end{tabular}

Table 3. Laboratory findings of Vietnamese study participants with diabetes

\begin{tabular}{|c|c|c|c|c|c|}
\hline & \multicolumn{2}{|c|}{ Median (range) } & \multicolumn{3}{|c|}{ Univariate analysis $^{\mathrm{a}}$} \\
\hline & $\begin{array}{c}\text { ED } \\
(n=113)\end{array}$ & $\begin{array}{c}\text { Non-ED } \\
(n=22)\end{array}$ & Crude OR & $95 \% \mathrm{Cl}$ & $p$ value \\
\hline Fasting blood glucose, $\mathrm{mg} / \mathrm{dL}$ & $135(77,531)$ & $138(76,260)$ & 1.00 & $0.99-1.01$ & 0.61 \\
\hline Hemoglobin A1c, \% & $7.9(5.5,14.2)$ & $7.8(5.4,12.9)$ & 1.06 & $0.84-1.33$ & 0.62 \\
\hline \multicolumn{6}{|l|}{ Serum lipid profile, $\mathrm{mg} / \mathrm{dL}$} \\
\hline Total cholesterol & $171(105,594)$ & $164(112,276)$ & 1.00 & $0.99-1.01$ & 0.62 \\
\hline HDL-cholesterol & $40(26,67)$ & $42(30,56)$ & 0.98 & $0.93-1.04$ & 0.55 \\
\hline LDL-cholesterol & $93(38,174)$ & $96(60,165)$ & 1.00 & $0.98-1.01$ & 0.54 \\
\hline Triglyceride & $188(61,2088)$ & $197(79,575)$ & 1.00 & $0.998-1.00$ & 0.52 \\
\hline \multicolumn{6}{|l|}{ Serum sex hormones } \\
\hline $\mathrm{LH}, \mathrm{mUl} / \mathrm{mL}$ & $4.41(1.43,19.39)$ & $3.60(2.11,11.06)$ & 1.07 & $0.88-1.30$ & 0.49 \\
\hline $\mathrm{FSH}, \mathrm{mUI} / \mathrm{mL}$ & $7.42(2.07,69.29)$ & $5.94(3.43,27.82)$ & 0.99 & $0.94-1.05$ & 0.80 \\
\hline Testosterone, $\mathrm{ng} / \mathrm{mL}$ & $4.01(0.60,8.55)$ & $4.07(2.21,15.0)$ & 0.85 & $0.66-1.08$ & 0.18 \\
\hline
\end{tabular}

Although the importance of routine screening for ED among patients with diabetes is widely recognized, a previous report from the United Kingdom points out a wide gap between recognition and clinical practice. ${ }^{19}$ It is not routine practice for Vietnamese diabetes specialists to assess ED. Patients are likewise culturally reluctant to discuss the matter. Our results raise two important clinical implications. First, the presence of peripheral neuropathy may be a key entry point for ED assessment. Second, the IIEF-5 questionnaire was shown to be easily applied even in busy outpatient clinics, and is highly accepted among patients.
Our study has 3 major methodological limitations. First, is the lack of validity testing of the IIEF-5 questions as translated into Vietnamese. Further investigation in collaboration with urologists to confirm the sensitivity and specificity of this tool in comparison with clinical diagnosis is required to identify the most suitable threshold for diagnostic cut-off.

Second, is the sampling method, which was via convenience sampling of a rather small group of patients visiting a tertiary hospital. We should be cautious in generalizing the obtained prevalence of ED to Vietnamese 
patients with diabetes in general. However, considering that a previous study in patients with diabetes in Vietnam reported a possibly high prevalence, the magnitude of the problem is certainly not negligible. Furthermore, even with our small sample size, we observed a strong association between ED and peripheral neuropathy.

Third, is the unexplained non-significant association between ED and previously known risk factors, including smoking, alcohol consumption, exercise and cardiovascular diseases. In some cases, the ORs seemed to trend toward the opposite direction, in comparison to previous reports, although these did not reach statistical significance. For the items on behavioral health, the conflicting results may be due in part to the cross-sectional nature of the study. Patients often quit regular alcohol intake and smoking after disease status worsens. This may yield weak associations between these factors and the outcome. For cardiovascular diseases, only a few cases were seen in the recruited patients. Most patients with these complications are commonly seen in cardiology or nephrology clinics.

\section{CONCLUSION}

ED is a major and common complication among men with type 2 diabetes. The presence of peripheral neuropathy and long duration of diabetes may be useful indicators of ED. Erectile dysfunction screening as routine clinical practice may be justified, so that adequate consultation and treatment can be offered.

\section{Acknowledgements}

The authors declare no conflict of interest. We conducted the present study in part as the Japan International Cooperation Agency's Partnership Program (Local Government Type), "Capacity building toward evidence-based medicine among health care professionals at University of Medicine and Pharmacy, Ho Chi Minh City and its related institutes."

\section{References}

1. Ho CC, Singam P, Hong GE, et al. Male sexual dysfunction in Asia. Asian J Androl. 2011;13(4):537-4.

2. Cheng JY, Ng EM, Chen RY, et al. Prevalence of erectile dysfunction in Asian populations: A meta-analysis. Int $\mathrm{J}$ Impot Res. 2007;19(3):229-44
3. Nicolosi A, Moreira ED Jr, Shirai M, et al. Epidemiology of erectile dysfunction in four countries: Cross-national study of the prevalence and correlates of erectile dysfunction. Urology. 2003;61(1):201-6.

4. Malavige LS, Levy JC. Erectile dysfunction in diabetes mellitus. J Sex Med. 2009;6(5):1232-47.

5. Ponholzer A, Temml C, Mock K, et al. Prevalence and risk factors for erectile dysfunction in 2869 men using a validated questionnaire. Eur Urol. 2005;47(1):80-5.

6. Phé V, Rouprêt M. Erectile dysfunction and diabetes: A review of the current evidence-based medicine and a synthesis of the main available therapies. Diabetes Metab. 2012;38(1):1-13.

7. Giang CHT, Nhu NT, Chuyen VL. Prevalence of erectile dysfunction of admitted patients more than 40 years old at the Department of Urology A, Binh Dan Hospital. Ho Chi Minh City Journal of Medicine. 2007;11:257-61. (In Vietnamese)

8. Viet PN, Tin PM, Duc NH, et al. Prevalence of erectile dysfunction in type 2 diabetic patients. Ho Chi Minh City Journal of Medicine. 2009;13:201-4. (In Vietnamese)

9. Rosen RC, Cappelleri JC, Smith MD, et al. Development and evaluation of an abridged, 5-item version of the International Index of Erectile Function (IIEF-5) as a diagnostic tool for erectile dysfunction. Int J Impot Res. 1999;11(6):319-26.

10. Yokokawa H, Khue NT, Goto A, et al. Diabetes control among Vietnamese patients in Ho Chi Minh city: An observational crosssectional study. International Electronic Journal of Health Education. 2010;13:1-13.

11. Cornblath DR. Diabetic neuropathy: Diagnostic methods. Adv Stud Med. 2004;4(8A):S650-61.

12. Perkins BA, Olaleye D, Zinman B, et al. Simple screening tests for peripheral neuropathy in the diabetes clinic. Diabetes Care. 2001;24(2):250-6.

13. Cho NH, Ahn CW, Park JY, et al. Prevalence of erectile dysfunction in Korean men with type 2 diabetes mellitus. Diabet Med. 2006;23(2):198-203.

14. Giugliano F, Maiorino M, Bellastella G, et al. Determinants of erectile dysfunction in type 2 diabetes. Int J Impot Res. 2010;22(3):204-9.

15. Yu LW, Kong AP, Tong PC, et al. Evaluation of erectile dysfunction and associated cardiovascular risk using structured questionnaires in Chinese type 2 diabetic men. Int J Androl. 2010;33(6):853-60.

16. Bacon CG, Hu FB, Giovannucci E, et al. Association of type and duration of diabetes with erectile dysfunction in a large cohort of men. Diabetes Care. 2002;25(8):1458-63.

17. Kalter-Leibovici O, Wainstein J, Ziv A, et al. Clinical, socioeconomic, and lifestyle parameters associated with erectile dysfunction among diabetic men. Diabetes Care. 2005;28(7):1739-44.

18. Sasaki H, Yamasaki H, Ogawa K, et al. Prevalence and risk factors for erectile dysfunction in Japanese diabetics. Diabetes Res Clin Pract. 2005;70(1):81-9.

19. Grant PS, Lipscomb D. How often do we ask about erectile dysfunction in the diabetes review clinic? Development of a neuropathy screening tool. Acta Diabetol. 2009;46:285-90.

Articles and any other material published in the JAFES represent the work of the author(s) and should not be construed to reflect the opinions of the Editors or the Publisher. Authors are required to accomplish, sign and submit scanned copies of the JAFES Declaration: that the article represents original material, that is not being considered for publication or has not been published or accepted for publication elsewhere. Consent forms, as appropriate, have been secured for the publication of information about patients; otherwise, authors declared that all means have been exhausted for securing such consent. The authors have signed disclosures that there are no financial or other relationships that might lead to a conflict of interest. All authors are required to submit Authorship Certifications that the manuscript has been read and approved by all authors, and that the requirements for authorship have been met by each author. 